\title{
Carcinoma hepatocelular na cirrose criptogênica: experiência de um centro
}

\section{Hepatocellular carcinoma in cryptogenic cirrhosis: a single center experience}

\author{
Marcya Moanna Gonçalves Cruz Macedo ${ }^{1}$. Paulo Everton Garcia Costa ${ }^{1}$. Bartolomeu Feitosa Neto ${ }^{1}$. Antônio \\ Brazil Viana Junior ${ }^{1}$. Tacilla Hanny de Souza Andrade ${ }^{2}$. Maurício Yukio Ogawa ${ }^{3}$. José Huygens Parente \\ Garcia $^{1}$ \\ 1 Hospital Universitário Walter Cantídio (HUWC), Fortaleza, Ceará, Brasil. 2 Universidade Estadual do Ceará (UECE), Fortaleza, \\ Ceará, Brasil. 3 Universidade Federal do Ceará (UFC), Fortaleza, Ceará, Brasil.
}

\section{RESUMO}

Objetivos: Avaliar as características clínicas de pacientes transplantados por carcinoma Hepatocelular (CHC) por cirrose Criptogênica e Vírus da Hepatite C. Métodos: Foram analisados, retrospectivamente, 45 pacientes entre 2003 a 2015. Dentre eles, 19 correspondiam a pacientes que transplantaram por etiologia indefinida da cirrose e CHC e 26 com infecção do vírus da Hepatite $\mathrm{C}(\mathrm{VHC})+\mathrm{CHC}$. Foram excluídos 4 pacientes, pois descobriu-se outra etiologia para a cirrose após histopatológico do explante, sendo utilizado teste qui-quadrado para análise. Resultados: 10 variáveis foram analisadas entre os grupos, evidenciando que a Bilirrubina ( $p<0,035)$, albumina ( $<<0,03)$, número de nódulos ( $<<0,026)$, CHILD $(p<0,003)$ e INR $(p<0,035)$ tiveram diferença significativa entre os dois grupos, sendo mais anormal naqueles com cirrose criptogênica. Aproximadamente $21 \%$ dos pacientes com cirrose criptogênica apresentam evidência para outras patologias. Conclusão: Muito esteato hepatites não alcoólicas e doença gordurosa não alcoólica do fígado são consideradas criptogênicas, sendo o tempo de permanência na fila maior que os pacientes com VHC; Transplantes por CHC por cirrose criptogênica são acompanhados por piores resultados laboratoriais e maior número de nódulos acometidos quando comparados com aqueles por VHC.

Palavras-chave: Cirrose hepática. Transplante de fígado. Hepatopatia gordurosa não alcoólica.

\section{ABSTRACT}

Objectives: To evaluate the clinical characteristics of patients transplanted by Hepatocellular carcinoma (HCC) by Cryptogenic cirrhosis and Hepatitis C virus. Method: A total of 45 patients were retrospectively analyzed between 2003 and 2015. Of these, 19 were patients who transplanted under the undetermined etiology of cirrhosis and $\mathrm{CHC}$ and 26 with Hepatitis $\mathrm{C}$ virus $(\mathrm{HCV})+\mathrm{CHC}$ infection. Four patients were excluded because another etiology for cirrhosis after histopathology of the explant was discovered, using a chi-square test for analysis. Results: Ten variables were analyzed between groups, including bilirubin $(\mathrm{P}<0.035)$, albumin $(\mathrm{P}<0.03)$, the number of nodes $(\mathrm{P}<0.026)$, Child-Pugh rating $(\mathrm{P}<0.003)$ and INR $(\mathrm{P}<0.035)$, and there were significant differences among the groups that were more abnormal in patients with cryptogenic cirrhosis. Approximately $21 \%$ of patients with cryptogenic cirrhosis showed evidence of other pathologies. Conclusion: Many heteroatons non-alcoholic hepatitis and non-alcoholic fatty liver disease are considered cryptogenic, with the length of stay in the queue being greater than the HCV patients; Transplantation for $\mathrm{CHC}$ due to cryptogenic cirrhosis is accompanied by worse laboratory results and a greater number of nodules involved when compared with those due to HCV.

Keywords: Hepatic cirrhosis. Liver transplantation. Non-alcoholic fatty liver disease.

Autor correspondente: Maurício Yukio Ogawa, Rua Coronel Nunes de Melo, 1315, Rodolfo Teófilo, Fortaleza, Ceará. CEP: 60430-160. Telefone: +55 85 99701-3486. E-mail: yukiogawa@gmail.com

Conflito de interesses: Não há qualquer conflito de interesses por parte de qualquer um dos autores.

Recebido em: 05 Mar 2018; Revisado em: 03 Out 2018; Aceito em: 08 Abr 2019. 


\section{INTRODUÇÃO}

O Carcinoma Hepatocelular (CHC) é o tumor maligno primário mais comum do fígado. Estima-se pela Organização Mundial de Saúde, 782 mil pessoas afetadas e 746 mil morreram no mundo em 2012. Sua incidência é mais alta no Leste e Sudeste da Ásia, que está relacionado a Hepatite Crônica do Vírus da Hepatite B (VHB) nessas regiões. Na verdade, ela é a segunda causa mais comum de morte por câncer em todo o mundo. ${ }^{1,2,3}$ Normalmente o CHC surge em um fígado cirrótico, e também é atualmente a principal causa de mortalidade nestes pacientes, independente da etiologia.

O diagnóstico de cirrose Criptogênica é considerado de exclusão, após descartar as etiologias virais (Hepatite B, C, D), medicamentosas, metabólicas (Doença de Wilson, Hemocromatose, Esteato Hepatite não alcóolica - NASH), imunes (Hepatite auto - imune, Cirrose biliar primaria, Colangite Esclerosante), alcoólica (consumo superior de álcool: $140 \mathrm{~g} / \mathrm{semana}$ homem e $70 \mathrm{~g} /$ semana mulher), sendo confirmado por histopatológico de explante. ${ }^{4}$

A Esteatose Hepática não alcoólica (EHNA), cada vez mais parece ser uma causa de CHC por causa da recente epidemia global de obesidade, podendo progredir para cirrose e/ou carcinoma hepatocelular. Não necessariamente necessitando do aparecimento de cirrose para desenvolver o câncer. A EHNA é responsável por aproximadamente 13\% de todos os casos de carcinoma hepatocelular nos Estados Unidos, e por $2 \%$ dos casos no Japão. No entanto, assim que a cirrose desenvolve-se durante o curso da doença, as características típicas, tais como esteatose e inflamação, desaparecem, e o diagnóstico histopatológico do explante fica mais difícil. ${ }^{5,6}$

Por conseguinte, com a exclusão de HBV, HCV, álcool, e outras etiologias conhecidas (doenças auto-imunes, genéticas), a maioria dos casos de cirrose por NASH são consideradas como criptogênicas, a menos que o paciente seja portador de síndrome metabólica, tenha sinais de aumento de ecogenicidade hepática em exames de imagem, antes do aparecimento da cirrose, como a estatose hepática, ou mesmo biópsia hepática compatível com NASH. 3,6,7

Em virtude do pouco relato na literatura sobre esse assunto, o presente estudo, tem como objetivo descrever as características clínicas específicas de pacientes com Cirrose Criptogência e $\mathrm{CHC}$ e descrever os fatores associados envolvidos na patogênese do $\mathrm{CHC}$ nestes pacientes.

\section{MATERIAIS E MÉTODOS}

No período de 13 anos de funcionamento do serviço de Transplante Hepático do Hospital Universitário Walter Cantídio (HUWC-UFC), Fortaleza - Ceará, Brasil, de 2003-2015, foram realizados 250 transplantes de fígado por Cirrose Hepática e Carcinoma Hepatocelular. Dentre esses, 19 pacientes não se tinha uma etiologia definida da cirrose no momento do diagnóstico, os quais foram comparados com outro grupo de 26 pacientes com Cirrose Hepática por
Vírus Hepatite C (VHC) e CHC ( VHC + CHC), que foram selecionados de forma aleatória nesse mesmo período, para avaliar o perfil demográfico entre esses grupos.

O diagnóstico de cirrose foi estabelecido por achados clínicos, como ascite, encefalopatia; laboratoriais, como plaquetopenia, albumina e INR alterados; exames de imagem evidenciando hipertensão portal, alteração de ecogenicidade hepática e/ou endoscopia com varizes esofágicas.

O diagnóstico de $\mathrm{CHC}$ em todos os pacientes, foi estabelecido usando níveis de Alfa-fetoproteína e exame radiológico: Ultrassonografia (USG), Tomografia Computadorizada (TC) e/ou Ressonância Nuclear Magnética (RNM) com contraste venoso, compatível com essa patologia, com captação precoce do contraste pela lesão na fase arterial, e clareamento rápido em fase de equilíbrio, conhecido como Wash-out. Sendo realizado também, TC de tórax e Cintilografia Óssea para avaliar alguma contraindicação ao Transplante, em que todos os pacientes preenchiam os critérios de Milão ${ }^{8}$ (nódulo único de até $5 \mathrm{~cm}$ ou até 3 nódulos de até $3 \mathrm{~cm}$, sem invasão vascular ou metástase a distância) e estavam aptos ao transplante.

O diagnóstico de Cirrose Criptogênica, foi estabelecido quando sorologias para vírus B (HBSAG), vírus C (anti$\mathrm{HCV}$ ) forem negativas, anticorpo anti-núcleo (FAN), antimúsculo liso, anti-mitocôndria, anti-LKM1, ceruloplasmina, Índice Saturação Transferrina (IST), alfa-1antitripsina não reagentes. E para paciente etilistas, consumo de álcool inferior a $20 \mathrm{~g} / \mathrm{dia}$.

Foram analisados: sexo, idade, níveis de alfa-fetoproteína antes do transplante, Child-Pugh score for cirrhosis mortality (CHILD), ${ }^{9}$ Model for end-stage liver disease (MELD), ${ }^{9}$ tempo de seguimento até a realização do procedimento cirúrgico, hábitos de vida, tipagem sanguínea, critérios para síndrome metabólica e exames laboratoriais como: Tempo de protombina (TAP/INR), Blirrubina Total e frações (BTF), albumina, aminotransferases (TGO, TGP, FA, GGT), Hemoglobina, creatinina, Sódio, Glicemia de Jejum e Índice de Massa corporal (IMC), a fim de traçar o perfil de cada grupo.

O teste qui-quadrado foi usado para avaliar a associação entre essas variáveis. O Statistical Package for the Social Sciences (SPSS) para Windows, versão 22.0, foi utilizado para essa análise e o valor de $\mathrm{P}<0.05$ foi considerado estatisticamente significativo.

Sendo analisado também o explante desses 45 pacientes que realizaram transplante hepático, para avaliar demais variáveis como a quantidade dos nódulos, tamanho dos mesmos, presença de invasão vascular, achados no explante que evidenciasse outra causa para etiologia da cirrose, que não a criptogênica.

Houve aprovação do Comitê de Ética e Pesquisa do Hospital Universitário Walter Cantídio para realização do estudo. 


\section{RESULTADOS}

Ao todo foram selecionados 45 pacientes transplantados por CHC durante um período compreendido de 2003 a 2015, contudo apenas 41 foram utilizados para análise, pois dos 19 pacientes em que a etiologia creditava ser criptogênica, 4 foram desconsiderados, pois no explante foi identificado que 2 eram portadores de NASH, 1 de hepatite autoimune e 1 de cirrose biliar primária, restando, pois, 15 pacientes.

Já os 26 restantes tinham como etiologia vírus da hepatite $\mathrm{C}$ (VHC) (Figura 1).

Figura 1. Casuística analisada.

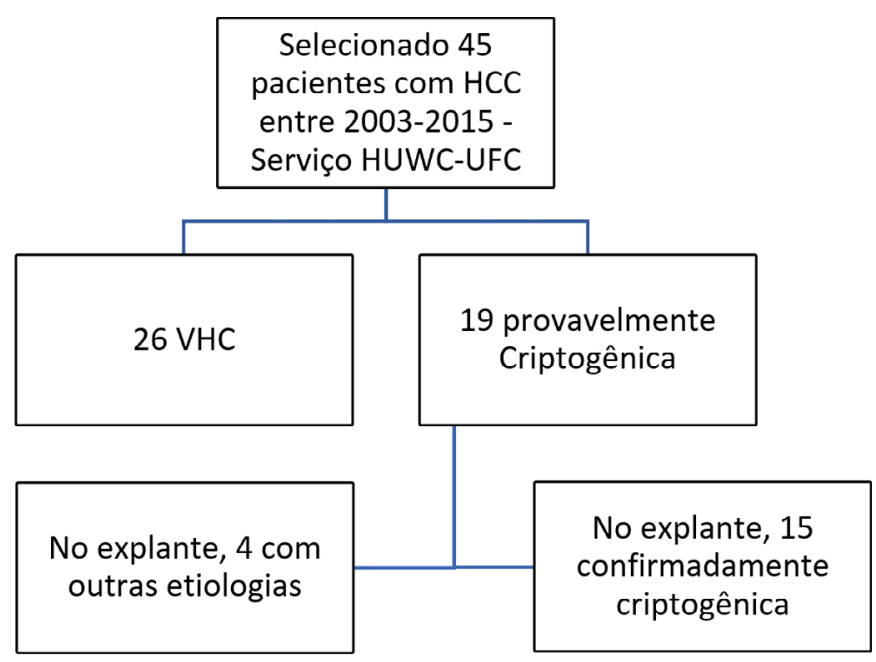

Dentre todos os aspectos clínicos e laboratoriais analisados, os fatores que tiveram significância $(\mathrm{p}<0,05)$ foram 5 ao todo. $\mathrm{O}$ primeiro deles foi CHILD ( $\mathrm{p}<0,003)$, sendo a maior parte dos pacientes com vírus da hepatite CHILD A $(65,4 \%)$, enquanto a maioria dos com Criptogênica CHILD B e C (80\%).

A albumina foi outra $(\mathrm{p}<0,03)$, sendo visto que a maior parte dos pacientes com criptogênica $(84,6 \%)$ apresentavam albumina abaixo de 3,5 , enquanto os com VHC, a maioria $(66,7 \%)$ apresentavam albumina acima de 3,5.

O terceiro foi o número de nódulos $(\mathrm{p}<0,026)$, sendo visto que os pacientes com VHC apresentam nódulo único na maioria dos casos $(69,2 \%)$, enquanto os com criptogênica apresentam mais de um nódulo $66,7 \%$.

Os outros dois fatores significantes foram bilirrubina $(\mathrm{p}<0,03)$, no qual maioria dos casos com VHC apresentavam valores normais $(61,5 \%)$, enquanto os com criptogênica apresentavam alterações tendo $40 \%$ dos pacientes valores entre 1,1 e 3 e 46,7 $\%$ com valores acima de 3,1 .

E por fim, o INR $(\mathrm{p}<0,035)$ foi inferior a 1,5 na maior parte dos pacientes com VHC $(82,6 \%)$, enquanto nos casos com criptogênica foi distribuído igualmente entre os que tinham valores inferiores a $1,5(50 \%)$ e os que tinham valores superiores a $1,5(50 \%)$.
Tais resultados estão representados na Tabela 1 juntamente a outros dados analisados sem significância estatística.

Tabela 1. Aspectos clínico-laboratoriais do grupo de pacientes com cirrose criptogênica $\mathrm{x}$ pacientes portadores de vírus da hepatite $\mathrm{C}$.

\begin{tabular}{lccc}
\hline Variáveis & CRIPTO (n=15) & VHC (n=26) & P \\
\hline Sexo masculino (\%) & $9(60)$ & $19(73)$ & 0,386 \\
Tabagismo (\%) & $8(57)$ & $9(34)$ & 0,169 \\
Etilismo(\%) & $5(36)$ & $15(57)$ & 0,185 \\
Albumina <3,5(\%) & $11(84)$ & $8(33)$ & 0,003 \\
Número nódulos - & $10(66)$ & $8(30)$ & 0,026 \\
multi (\%) & & & \\
Tamanho nódulo & $8(53)$ & $10(38)$ & 0,219 \\
$<3$ cm (\%) & & & \\
Bilirrubina >3,1 (\%) & $7(46)$ & $2(7,7)$ & 0,003 \\
TGP ATE 3XLSN (\%) & $7(46)$ & $16(61)$ & 0,250 \\
TGO ATE 3XLSN (\%) & $11(78)$ & $20(76)$ & 0,171 \\
INR>=1,5(\%) & $7(50)$ & $4(17)$ & 0,035 \\
CHILD C(\%) & $6(40)$ & $1(4)$ & 0,003 \\
MELD <20(\%) & $12(80)$ & $19(73)$ & 0,712 \\
SD metabólica (\%) & $4(27)$ & $5(24)$ & 0,845 \\
\hline
\end{tabular}

\section{DISCUSSÃO}

Temos a ciência de que o CHC tem sido cada vez mais prevalente em países ocidentais associada principalmente à cirrose, sendo a hepatite $\mathrm{C}$ a causa mais comum de cirrose hepática no Brasil $^{10}$ e paralelo a isso o fato de que o CHC criptogênico possui ainda poucos estudos quanto as manifestações clínicas e o prognóstico, sendo uma doença comumente diagnosticada já em estágios avançados,,$^{3}$ o que é corroborado por nosso estudo na medida em que os parâmetros, como níveis séricos de albumina e bilirrubina, encontram-se em níveis mais alterados nesses pacientes. A cirrose pelo vírus da hepatite $\mathrm{C}$ e a criptogência são as duas principais indicações de transplante hepático. ${ }^{11}$

Dos 250 pacientes transplantados com Cirrose e CHC nestes 13 anos em nosso serviço, 14 pacientes foram confirmados com CHC-Criptogênica totalizando uma prevalência de $5,6 \%$, a qual foi compatível com a literatura, como observa-se em um estudo realizado no Japão no ano de 1998 com uma prevalência de $5 \% .^{12}$

Com a avaliação do explante, pode-se notar que dos 19 pacientes que eram tidos como Criptogênico, 21\% tinham uma causa definida para a cirrose, que não a criptogênica. Levando-se em conta que na cirrose avançada os achados histopatológicos podem ficar menos evidentes, ${ }^{13}$ esta prevalência da cirrose criptogênica pode estar superestimada.

O presente estudo diverge de uma parte da literatura médica atual, na medida em que esta mostra o perfil dos pacientes 
com CHC criptogênica com várias comorbidades, como idade avançada e acompanhando os fatores de risco para a síndrome metabólica, e outros dados, como, por exemplo, nódulo único. ${ }^{7}$

No nosso estudo observou-se que os pacientes com Cirrose criptogênica, permaneciam em fila de transplante por mais tempo que os pacientes com VHC (Tabela 2), pois no momento do diagnóstico os com Hepatite já fechavam critérios para transplante, sendo transplantado como situação especial em menos de 1 ano (92,3\%), a maioria nos primeiros 6 meses. Diferente dos pacientes com Criptogênica, em que $40 \%$ transplantou após 1 ano de seguimento da doença. Observando assim piores níveis laboratorias e maior quantidade de nódulos no achado do explante.

Tabela 2. Tempo de seguimento até transplante hepático.

\begin{tabular}{llll}
\hline $\begin{array}{l}\text { Tempo seguimento } \\
\text { até TX hepático }\end{array}$ & VHC & Cripto* & P \\
\hline$<1$ ano & $24(92,3 \%)$ & $7(46,6 \%)$ & \\
$>1$ ano & $2(7,7 \%)$ & $6(40,0 \%)$ & 0,005 \\
Total & $26(100 \%)$ & $13(86,6 \%)$ & \\
\hline
\end{tabular}

*Obs: 2 pacientes com criptogênica não foram incluídos nesse quesito, pois não foi possível localizar os dados referente aos mesmos.

Um questionamento que é aventado por nossa pesquisa é se realmente não há diferença significativa na sobrevida a longo prazo entre pacientes com HCC criptogênico e virais/ álcoolico ${ }^{14}$ ou se existe uma melhor sobrevida nos pacientes criptogênicos, ${ }^{15}$ pois foi-se visto que parâmetros, como CHILD e níveis séricos de albumina, encontram-se em melhor estágio

\section{REFERÊNCIAS}

1. Kumaran V. Role of liver transplantation for hepatocellular carcinoma. J Clin Exp Hepatol. 2014;4:S97-103.

2. Goodman ZD. The impact of obesity on liver histology. 2014;18:22042.

3. Pang TC, Lam VW. Surgical management of hepatocellular carcinoma. World J Hepatol. 2015;7(2):245-52.

4. Caldwell S. Cryptogenic cirrhosis: what are we missing? Curr Gastroenterol Rep. 2010;12(1):40-8.

5. Rao PN. Nodule in liver: investigations, differential diagnosis and follow-up. J Clin Exp Hepatol. 2014;4:S57-62.

6. Cheng AL, Amarapurkar D, Chao Y, Chen PJ, Geschwind JF, Goh $\mathrm{KL}$, et al. Re-evaluating transarterial chemoembolization for the treatment of hepatocellular carcinoma: consensus recommendations and review by an international expert panel. Liver Int. 2014;34(2):17483.

7. Yin W, Zhao Y, Ji YJ, Tong LP, Liu Y, He SX, et al. Serum/plasma microRNAs as biomarkers for HBV-related hepatocellular carcinoma in China. Biomed Res Int. 2015;2015(965185,):1-8. nos pacientes com Vírus da Hepatite C (VHC) em comparação com os criptogênicos. Sendo necessário estudos posteriores para identificar se existe, portanto, alguma mudança na sobrevida destes.

Um viés que poderia ser considerado em nosso estudo, seria o $\mathrm{n}$ pequeno de pacientes com hepatite $\mathrm{C}$, e a forma aleatória de seleção dos mesmos. Afim de comparar com a população de cirróticos criptogênicos.

Não foi encontrado uma justificativa do porquê de os pacientes criptogênicos passarem mais tempo em fila de transplante, ficando com sua doença cada vez mais avançada. Possivelmente o Vírus C por ser mais agressivo, já se descobre a doença com nódulo transplantável $(2-3 \mathrm{~cm})$, conseguindo o procedimento cirúrgico em até 6 meses.

A cirrose criptogênica, mais indolente, demora para entrar em critério para transplante, mas quando transplanta já encontra-se em situação mais delicada, com a função hepática bem mais prejudicada. Consequentemente, a sobrevida destes pós-transplante hepático deve ser inferior comparado ao Vírus C, porém mais estudos devem ser realizados para avaliar essa população muito pouco estudada mundialmente.

$\mathrm{O}$ presente estudo evidencia que em pacientes com $\mathrm{CHC}$ existem diferenças, principalmente nos exames laboratoriais, entre o grupo portador de cirrose criptogênica e de cirrose por vírus da hepatite $\mathrm{C}$. No geral, os pacientes com cirrose criptogênica apresentaram piores exames laboratoriais do que aqueles com VHC, sendo questionado pelos autores do presente trabalho se existe diferença na mortalidade destes pacientes.

8. Zhu Z. Milan criteria and its expansions in liver transplantation for hepatocellular carcinoma. HepatoBiliary Surg Nutr. 2016;5(6):498502.

9. Durand F, Valla D. Assessment of prognosis of cirrhosis. Semin Liver Dis. 2008;28(1):110-22.

10. Kumar A, Acharya SK, Singh SP, Saraswat VA, Arora A, Duseja A, et al. The Indian National Association for Study of the Liver (INASL) consensus on prevention, diagnosis and management of hepatocellular carcinoma in India: the puri recommendations. J Clin Exp Hepatol. 2014;4(Supl 3):S3-26.

11. Viana RB. Causa de óbito tardio em transplantados de fígado. Rev Assoc Med Bras. 2003;49(2):177-80.

12. Kasai K, Kuroda H, Suzuki K. [Adjuvant therapy after treatment of hepatocellular carcinoma]. Nihon Shokakibyo Gakkai Zasshi. 2008;105(6):787-94. Japanese.

13. Lida VH, Silva TJ, Silva AS, Silva LF, Alves VA. Cirrose hepática: aspectos morfológicos relacionados às suas possíveis complições. Um estudo centrado em necropsias. J Bras Patol Med Lab. 2005;41(1):29-36. 
14. Ferlay J, Shin HR, Bray F, Forman D, Mathers C, Parkin DM. Estimates of worldwide burden of cancer in 2008: GLOBOCAN 2008. Int J Cancer. 2010;127(12):2893-917.
15. Yang JD, Roberts LR. Hepatocellular carcinoma: a global view. 2014;7(8):448-58.

\section{Como citar:}

Macedo MM, Costa PE, Feitosa B Neto, Viana AB Junior, Andrade TH, Ogawa MY, et al. Carcinoma hepatocelular na cirrose criptogênica: experiência de um centro. Rev Med UFC. 2019 out-dez;59(4):51-55. 